post-traumatic stress disorder (17.5\%), and diabetes (14.8\%). During the first observed and treated MDE (mean \pm SD duration: $14.7 \pm 8.6$ months), patients received a mean of $1.6 \pm 1.0$ LOTs, with $36.5 \%$ and $14.6 \%$ of patients receiving $\geq 2$ and $\geq 3$ LOTs, respectively; $0.8 \%$ of patients received $\geq 6$ LOTs. The most commonly observed therapies were SSRI monotherapy $(58.9 \%)$ followed by SNRI monotherapy (8.8\%) in LOT1; SSRI monotherapy followed by AD augmented with anticonvulsants in LOT2 (SSRI monotherapy: 48.7\%; $\mathrm{AD}$ augmentation with anticonvulsants:12.1\%) and LOT3 (SSRI monotherapy: $43.5 \%$; AD augmentation with anticonvulsants:15.0\%).

Conclusions. This study used an episodic approach to evaluate the current standard of care among veterans with MDD. During the first observed and treated MDE, about one in seven veterans received $\geq 3$ LOTs, suggesting presence of treatment-resistant MDD. Monotherapy with SSRIs or SNRIs and combination therapies of $\mathrm{AD}$ with anticonvulsants were the most common therapies in the first three LOTs.

Funding. Janssen Scientific Affairs, LLC

\section{Ornithine Transcarbamylase Deficiency Presenting with Symptoms of Mania in a Young Adult Male}

Jonathan Pentz, MD, Blessing Igboeli, MD and Julie Niedermier, MD

The Ohio State University, Columbus, OH, USA

Presenting Author: Jonathan Pentz

\begin{abstract}
Study Objective. The purpose of this case study is to review the clinical presentation and medical workup of a young adult male presenting with acute behavior changes in the setting of undiagnosed ornithine transcarbamylase deficiency (OTCD)

Method. This case study involves a 19-year-old male with a psychiatric history of depression and one previous suicide attempt, who presented to a large midwestern university hospital emergency department after being found by police naked in a neighbor's yard. He displayed manic signs and symptoms, including euphoria, lack of sleep for five days, and attempting to purchase a new car and three large screen TVs. Family reported the patient uncharacteristically announced three weeks earlier that he was vegetarian and stopped eating his frequent customary cheeseburgers. Due to increased anxiety and inability to sleep, the patient received lorazepam $2 \mathrm{mg}$ in the emergency department. Upon transfer to the psychiatric unit, therapy was initiated with aripiprazole $5 \mathrm{mg}$ daily and valproate $1000 \mathrm{mg}$ nightly on Day 1 of treatment. The patient refused medications on hospital Day 2 , then received this combination again on Day 3. The next morning, the patient complained of lethargy, headache, nausea, and vomiting.

Results. The patient's ammonia level was found to be 204 micro$\mathrm{mol} / \mathrm{L}$ with ALT and AST of 714 and $647 \mathrm{IU} / \mathrm{L}$ respectively. Tests
\end{abstract}

for infectious hepatitis were negative. Medical consultation recommended discontinuation of current medications, vigorous hydration, and further work up. On further investigation, the patient was found to have low plasma citrulline level of 8 micro$\mathrm{mol} / \mathrm{L}$, undetectable plasma arginine, and high urinary orotic acid. The laboratory data showed a biochemical phenotype consistent with a diagnosis of partial OTCD, an X-linked urea cycle disorder resulting in toxic hyperammonemia. The patient was treated with a low protein diet modification as well as a combination of sodium benzoate and sodium phenylbutyrate to reduce serum ammonia concentration. With treatment the patient's laboratory values normalized, and mental status improved.

Conclusions. In conclusion, partial ornithine transcarbamylase deficiency may manifest with psychiatric symptoms in early adulthood. In young patients with elevated ammonia and mental status change, OTCD is an important diagnosis to consider, as it is the most common inherited cause of hyperammonemia.

\section{The Effectiveness of De-Escalation Techniques as Compared to Physical Restraint/Seclusion on Inpatient Psychiatric Units: A Quantitative Systematic Review}

\author{
Shavar Chase, DNP, PMHNP-BC
}

Rutgers University, Newark, NJ, USA

Presenting Author: Shavar Chase

\begin{abstract}
Background. Restraint and seclusion were considered a form of treatment but consistently has led to physical and mental injuries to staff and patients. De-escalation has been viewed as a safer option. Understanding which intervention yields decreased injuries, aggression and violence will guide policy and inform practice.

Objectives. To identify which intervention leads to decreased physical and psychological injury to patients and staff.

Methods. The frequency of physical injuries to patients and staff from aggressive patients; frequency of psychological injuries to patients and staff from violent, aggressive incidents; frequency of violence, agitation and aggression; competence of staff at managing aggression and violence were evaluated.

Results. Fourteen studies were included in this review. There are many forms of de-escalation. Studies where techniques were taught to staff, the intervention was effective in decreasing injury in approximately half the studies. De-escalation techniques taught to patients decreased injury in $100 \%$ of the studies included in this review.

Conclusion. Consensus on which intervention works best could not be reached, nor is there overwhelming evidence for a particular type of de-escalation better suited for decreasing aggression and violence. Caution should be exercised when choosing a de-escalation technique for implementation in institutions due to lack of regulating agencies that inform practice and standards. In
\end{abstract}


addition, the literature lacks best practices for de-escalation techniques backed by evidence. Restraint and seclusion should be used as a last resort due to inherent risk associated with the intervention.

\section{Overcoming the Challenges of the Mental Health Care System in United States in the Aftermath of COVID-19}

\author{
Amir Radfar, MD, MPH, MSc, DHSc ${ }^{1}$, \\ Maria Mercedes Ferreira Caceres, $M D^{2}$, \\ Juan Pablo Sosa, MD $^{3}$ and Irina Filip, MD, DHSc ${ }^{4}$
}

\begin{abstract}
${ }^{1}$ University of Central Florida, Assistant Professor of Medical Education, Orlando, FL USA, ${ }^{2}$ Universidad Autónoma de Bucaramanga, Epidemiologist, Santander, Colombia, ${ }^{3}$ Universidad Nacional de Tucumán, San Miguel de Tucumán, Argentina, and ${ }^{4}$ Western University of Health Sciences, Assistant Professor of Clinical Psychiatry, Pomona, CA, USA
\end{abstract}

Presenting Author: Maria Mercedes Ferreira Caceres, Juan Pablo Sosa shortages, fragmented care, insufficient mental health care policies and insufficient education and awareness about mental illness become more prominent during the COVID-19 pandemic era. Conclusions. The impact of COVID-19 on mental health is alarming, which affects public health and has made the health care system more vulnerable. Pandemic events not only cause acute negative impact, they also result in long-lasting health problems, isolation and stigma. The COVID-19 pandemic threatens the mental health of the population and its long-term consequences can lead to a secondary pandemic. The outcomes of the COVID-19 pandemic on mental health emphasize the need for policies and strategies to support and strengthen a concerted effort to address its burden on the US mental health care system. In addition, it magnifies the need for high quality and well-funded research for future pandemics.

\section{Methylphenidate-Induced Chorea Due to Possible Cytochrome P450 Metabolism Heterogeneity - A Rare Case}

Sree Latha Krishna Jadapalle, MD ${ }^{1}$, Edwin McCray, BS $^{2}$, John Azat Masoud, BS $^{2}$ and Michael W. Kortz, BS $^{3}$

Study Objective(s). The impact of pandemic events such as the coronavirus (COVID-19) pandemic led to an economic crisis worldwide as well as an increase of mental health problems. In the United States, the gaps in the mental health care system struggle to meet the needs of vulnerable populations and have caused a major public health problem. We aim to increase the awareness of health care professionals, psychiatrists, and policy makers regarding failures and gaps in the mental health care system and suggest new ideas to overcome the growing burden of mental disorders. Method. We utilized data from PubMed, Science Direct, Cochrane, Embase and Clinicaltrials.gov databases to analyze available information on the US mental health system. We included any relevant articles addressing the prevalence of mental diseases, disparities and the gaps for an accessible and affordable mental health system, as well as the psychological impact of COVID-19 pandemic.

Keywords. 'COVID-19', 'Coronavirus', 'SARS-CoV-2', 'mental health', 'Health, Mental' were used.

Results. Following scoping review of several studies we noticed that while prevalence of mental health problems in the US varies between states and socio-demographic groups, it is among the top 10 causes of premature death and disability in adults. We noticed that mental health problems are currently one of the costliest public health issues in the healthcare system. Tracking Poll from one of the studies in our scoping review suggested that financial inequities are magnified by the COVID-19 pandemic and that psychological distress was substantially larger among respondents with lower income (33\%), Hispanics (28\%) and Blacks (26\%). Furthermore, in another poll $62 \%$ of US population are shown to be anxious due to COVID-19. We observed that the prevalence of reported symptoms of psychological distress among US adults increased when compared to 2018. Common barriers such as failure of accessibility, insufficient funding, insufficient psychiatric beds, limited insurance access and economic burden, clinician

${ }^{1}$ Campbell University School of Osteopathic Medicine, Assistant Professor of Psychiatry, Lillington, NC, USA and Cape Fear Valley Medical Center, Fayetteville, NC, USA, ${ }^{2}$ Campbell University School of Osteopathic Medicine, Lillington, NC, USA, and ${ }^{3}$ University of Colorado, Department of Neurosurgery, Aurora, CO, USA

Presenting Author: Sree Latha Krishna Jadapalle
Abstract
Background. Chorea is defined as a hyperactive movement disor- der associated with involuntary, quick, and unpredictable muscle contractions of the limbs, face, and trunk. The unpredictable nature of these movements includes variation in speed, timing, and direc- tion of movement. A wide variety of medications, medical condi- tions and illicit drugs have been associated with movement disorders. Examples include a multitude of antipsychotic induced movement disorders and dyskinesia related to dopaminergic agents, like levodopa and metoclopramide. Dyskinesias have been associated with psycho-stimulant use, such as methylphenidate. However, most cases reported were associated with large doses or chronic use. Aside from dyskinesia, methylphenidate is known to be associated with tic disorder, tremor, and muscle spasm. How- ever, this case reported is unlike any of the above described and involved the development of chorea after only 2 days of moderate doses of methylphenidate, in a patient on chronic methadone maintenance treatment, with successful arrest of symptoms follow- ing discontinuation of the methylphenidate.
Case Presentation. A 47-year-old female was admitted to our hospital after presenting to the emergency department with 1 week of violent flailing movements. The ballistic flailing movements started acutely after 2 days of initiating methylphe- nidate in addition to her chronic methadone treatment and 2 -week period of initiation of paroxetine. Lab work showed 